Bull. Korean Math. Soc. 46 (2009), No. 6, pp. 1221-1228

DOI 10.4134/BKMS.2009.46.6.1221

\title{
ON VOLUMES OF PARALLEL 2 $n$-HEDRON IN A LORENTZ VECTOR SPACE
}

\author{
Seongil Park, Byung Hak Kim, Jin Hyuk Choi, and Young Ok Lee
}

\begin{abstract}
In this paper we formulated the volume of parallel $2 n$-hedron spanned by timelike linearly independent vectors in the Lorentz vector space.
\end{abstract}

\section{Introduction}

The Lorentzian geometry is very deeply related to the study of general relativity and the theory of cosmology. And a good understanding of global Lorentzian geometry is essential for the development of singularity theory. Recent results and related works in the Lorentzian geometry can be found in the book [1] written by J. K. Beem, P. E. Ehrlich, and K. L. Easley.

The inner product or distance function in the Lorentzian geometry has many similarities with the counterparts in the Riemannian geometry but also has many differences with them. It is well known that the length of vectors, area of parallelogram spanned by two linearly independent vectors and the volume of parallel $2 n$-hedron spanned by $n$ linearly independent vectors in the Euclidean space are represented by the norms or the determinants. In Euclidean space, the volume $V^{n}\left(u_{1}, u_{2}, \ldots, u_{n}\right)$ of parallel $2 n$-hedron $(n \geq 3)$ spanned by linearly independent vectors $u_{1}, u_{2}, \ldots, u_{n}$ is given by $([2])$

$$
\left\{V^{n}\left(u_{1}, u_{2}, \ldots, u_{n}\right)\right\}^{2}=\left|\begin{array}{cccc}
\left\langle u_{1}, u_{1}\right\rangle & \left\langle u_{1}, u_{2}\right\rangle & \cdots & \left\langle u_{1}, u_{n}\right\rangle \\
\left\langle u_{2}, u_{1}\right\rangle & \left\langle u_{2}, u_{2}\right\rangle & \cdots & \left\langle u_{2}, u_{n}\right\rangle \\
\vdots & \vdots & \ddots & \vdots \\
\left\langle u_{n}, u_{1}\right\rangle & \left\langle u_{n}, u_{2}\right\rangle & \cdots & \left\langle u_{n}, u_{n}\right\rangle
\end{array}\right|
$$

where $\langle$,$\rangle is an Euclidean inner product and |A|$ is the determinant of $A$.

Let $V^{1}\left(u_{1}\right)$ be a volume(length) of linearly independent vector $u_{1}$ and let $V^{2}\left(u_{1}, u_{2}\right)$ be a volume(area) of the plane spanned by linearly independent vectors $u_{1}$ and $u_{2}$. Then we can easily see that $\left\{V^{1}\left(u_{1}\right)\right\}^{2}=\left\langle u_{1}, u_{1}\right\rangle=\left\|u_{1}\right\|^{2}$,

Received October 10, 2008.

2000 Mathematics Subject Classification. Primary 53B30, 53C50.

Key words and phrases. volumes, Lorentz vector space, timelike vector.

The first author was supported by the Research Grant 2006 of Kyung Hee University. 
$\left\{V^{2}\left(u_{1}, u_{2}\right)\right\}^{2}=\left\|u_{1} \times u_{2}\right\|^{2}$ and $\left\{V^{3}\left(u_{1}, u_{2}, u_{3}\right)\right\}^{2}=\left\{\left\langle u_{1}, u_{2} \times u_{3}\right\rangle\right\}^{2}$, where $u \times v$ is a vector product of $u$ and $v$. In this point of a view, the formulation of the volume $V_{1}^{n}\left(u_{1}, \ldots, u_{n}\right)$ of parallel $2 n$-hedron spanned by timelike linearly independent vectors $u_{1}, u_{2}, \ldots, u_{n}$ in the Lorentz vector space with a Lorentz product $b$ is meaningful and natural. In this paper, we obtain the formula of $V_{1}^{n}\left(u_{1}, \ldots, u_{n}\right)$ as

$$
\left\{V_{1}^{n}\left(u_{1}, u_{2}, \ldots, u_{n}\right)\right\}^{2}=\left|\begin{array}{cccc}
-b\left(u_{1}, u_{1}\right) & -b\left(u_{1}, u_{2}\right) & \cdots & -b\left(u_{1}, u_{n}\right) \\
-b\left(u_{2}, u_{1}\right) & -b\left(u_{2}, u_{2}\right) & \cdots & -b\left(u_{2}, u_{n}\right) \\
\vdots & \vdots & \ddots & \vdots \\
-b\left(u_{n}, u_{1}\right) & -b\left(u_{n}, u_{2}\right) & \cdots & -b\left(u_{n}, u_{n}\right)
\end{array}\right|
$$

in the Lorentz vector space $U_{1}^{n}$ with a Lorentz product $b$ (cf. Theorem 3.2).

\section{Semi-Riemannian spaces}

Now, we prepare some definitions and notations for later use, and summarize known results for our main theorem. Let $U$ be an $n$-dimensional real vector space and $b$ a symmetric bilinear form of $U \times U$ into $R$. The index $\nu$ of a symmetric bilinear form $b$ on $U$ is defined by the largest integer that is the dimension of a subspace $W$ of $U$ on which $\left.b\right|_{W}$ is negative definite. Hence, we can see that $0 \leq \nu \leq n, \nu=0$ if and only if bilinear form $b$ is positive definite, and $\nu=n$ if and only if $b$ is negative definite. In general, the symmetric bilinear form $b$ with index $\nu$ has the form

$$
b(v, w)=-\sum_{i=1}^{\nu} v_{i} w_{i}+\sum_{i=\nu+1}^{n} v_{i} w_{i} .
$$

The resulting semi-Riemannian space is denoted by $U_{\nu}^{n} . U_{\nu}^{n}$ is reduced to $U^{n}$ if $\nu=0$. If $0<\nu<n$, then $b$ is said to be indefinite. In particular, if $\nu=1$, then $b$ is called a Lorentz product and $U$ with such a symmetric bilinear form $b$ is called a Lorentz vector space.

A symmetric bilinear form $b$ on a vector space $U$ is called a scalar product if it is non-degenerate and semi-definite. Also, a symmetric bilinear form $b$ on $U$ is called an inner product if it is definite. A vector space $U$ is said to be a scalar product space, an inner product space or a Lorentz vector space provided that $U$ is furnished with a scalar product, an inner product or a Lorentz product respectively.

For a scalar product space $(U, b)$ equipped with a scalar product $b$, a vector $v$ in $U$ is said to be ([4])

$$
\begin{array}{rll}
\text { spacelike if } & b(v, v)>0 \quad \text { or } v=0, \\
\text { null if } & b(v, v)=0 \quad \text { and } v \neq 0, \\
\text { timelike if } & b(v, v)<0 . &
\end{array}
$$


Let $U$ be a Lorentz vector space with Lorentz product $b$ and $\mathcal{T}$ be the set of all timelike vectors in $U$. For $u \in \mathcal{T}$,

$$
C(u)=\{v \in \mathcal{T}: b(u, v)<0\}
$$

is called a timecone of $U$ containing $u$.

Vectors $v$ and $w$ in $U$ are said to be orthogonal if $b(v, w)=0$. Subsets $A$ and $B$ in $U$ are said to be orthogonal provided that $v$ and $w$ are orthogonal for any vectors $v$ in $A$ and $w$ in $B$. Orthogonal vectors $u, v$ or orthogonal subsets $A, B$ in $U$ are denoted by $u \perp v$ or $A \perp B$ respectively. For a subspace $W$ in $U$, the set $W^{\perp}$ consists of vectors in $U$, where every vector in $U$ is orthogonal to $W . W^{\perp}$ is called a $W$-perpendicular subspace of $U$. The following lemma is well known ([3]).

Lemma 2.1. Timelike vectors $v$ and $w$ in the Lorentz vector space are in the same timecone if and only if $b(v, w)<0$.

In a vector space with inner product, the Schwarz inequality is derived from definition of the angle $\theta$ between $v$ and $w$ as the unique number $0 \leq \theta \leq \pi$ such that $\cos \theta=\frac{b(v, w)}{\|v\|\|w\|}$. An analogus Lorentz case is as follows $([3])$.

Proposition 2.2. Let $v$ and $w$ be timelike vectors in a Lorentz vector space $U$. Then we have the following properties;

(1) $\|b(v, w)\| \geq\|v\|\|w\|$, where the equality holds if and only if $v$ and $w$ are collinear.

(2) If $v$ and $w$ are contained in the same timecone of $v$, then there is a unique number $\theta(\geq 0)$ such that

$$
b(v, w)=-\|v\|\|w\| \cosh \theta .
$$

In this case, $\theta$ is called the hyperbolic angle between $v$ and $w$.

From these properties, we can obtain the triangle inequality for Lorentzian geometry. Notice the fact that unlike the triangle inequality for Euclidean geometry, the norm of the addition of two timelike vectors is greater than the addition of the two norms of two timelike vectors considered.

Corollary 2.3 ([3]). If $v$ and $w$ are timelike vectors in the same timecone, then we have

$$
\|v\|+\|w\| \leq\|v+w\|
$$

where the equality holds if and only if $v$ and $w$ are collinear.

Next, we consider a property of a 3 -dimensional Lorentz vector space $U_{1}^{3}$ with a Lorentz product $b$. For any vectors $u=\left(u_{A}\right)=\left(u_{1}, u_{2}, u_{3}\right)$ and $v=$ $\left(v_{A}\right)=\left(v_{1}, v_{2}, v_{3}\right)$ in $U_{1}^{3}$, the Lorentz product is defined by $b(u, v)=-u_{1} v_{1}+$ $u_{2} v_{2}+u_{3} v_{3}$. Then a Lorentz cross product $u \times v$ is defined by

$$
u \times v=\left(-u_{2} v_{3}+u_{3} v_{2}, u_{3} v_{1}-u_{1} v_{3}, u_{1} v_{2}-u_{2} v_{1}\right)=\left|\begin{array}{ccc}
-i & j & k \\
u_{1} & u_{2} & u_{3} \\
v_{1} & v_{2} & v_{3}
\end{array}\right| \text {, }
$$


where $|A|$ is the determinant of $A$.

For the Lorentz cross product, the following properties are well-known ([3]).

Lemma 2.4. For $u, v, w, z \in U_{1}^{3}$, we have

(1) $u \times v=0$ if and only if $u$ and $v$ are collinear,

(2) $u \times v=-v \times u$,

(3) $b(u \times v, u)=b(u \times v, v)=0$,

(4) $b(u \times v, w)=b(v \times w, u)=\left|\begin{array}{ccc}u_{1} & u_{2} & u_{3} \\ v_{1} & v_{2} & v_{3} \\ w_{1} & w_{2} & w_{3}\end{array}\right|$, where $u=\left(u_{1}, u_{2}, u_{3}\right)$, $v=\left(v_{1}, v_{2}, v_{3}\right), w=\left(w_{1}, w_{2}, w_{3}\right)$,

(5) if $u$ or $v$ is timelike, then $u \times v$ is spacelike,

(6) $b(u \times v, w \times z)=b(u, z) b(v, w)-b(u, w) b(v, z)=\left|\begin{array}{ll}-b(u, z) & -b(u, w) \\ -b(v, z) & -b(v, w)\end{array}\right|$.

\section{Volumes of parallel $2 n$-hedron in a Lorentz vector space}

In this section, we study the volume $V_{1}^{n}\left(u_{1}, u_{2}, \ldots, u_{n}\right)$ of parallel $2 n$-hedron spanned by timelike linearly independent vectors $u_{1}, u_{2}, \ldots, u_{n}$ in a Lorentz vector space $U_{1}^{n}$. Then, using the Lemma 2.4, $V_{1}^{1}(u)$ and $V_{1}^{2}(u, v)$ are given by

$$
\left\{V_{1}^{1}(u)\right\}^{2}=\|u\|^{2}=-b(u, u)
$$

and

$$
\left\{V_{1}^{2}(u, v)\right\}^{2}=\|u \times v\|^{2}=-b(u \times v, u \times v)=\left|\begin{array}{ll}
-b(u, u) & -b(u, v) \\
-b(v, u) & -b(v, v)
\end{array}\right|,
$$

where $u, v$ are not in the same timecone.

For linearly independent timelike vectors $u, v$ and $w$, we assume that none of them are in the same timecone. Then the value of a symmetric bilinear form $b$ of any two vectors is non-negative by the Lemma 2.1. In this case, we can calculate $\left\{V_{1}^{3}(u, v, w)\right\}^{2}$ as follows:

Theorem 3.1. Let $u, v, w$ be linearly independent timelike vectors and none of them are in the same timecone. Then we have

$$
\left\{V_{1}^{3}(u, v, w)\right\}^{2}=\left|\begin{array}{lll}
-b(u, u) & -b(u, v) & -b(u, w) \\
-b(v, u) & -b(v, v) & -b(v, w) \\
-b(w, u) & -b(w, v) & -b(w, w)
\end{array}\right| .
$$

Proof. In a 3-dimensional Lorentz vector space $U_{1}^{3}$, the volume $V_{1}^{3}(u, v, w)$ of parallel 6 -hedron spanned by linearly independent timelike vectors $u=$ $\left(u_{1}, u_{2}, u_{3}\right), v=\left(v_{1}, v_{2}, v_{3}\right)$, and $w=\left(w_{1}, w_{2}, w_{3}\right)$ is

$$
b(u \times v, w)=\operatorname{det} A,
$$

where $A$ is the $3 \times 3$ matrix $\left(\begin{array}{ccc}u_{1} & u_{2} & u_{3} \\ v_{1} & v_{2} & v_{3} \\ w_{1} & w_{2} & w_{3}\end{array}\right)$. 
ON VOLUMES OF PARALLEL 2n-HEDRON IN A LORENTZ VECTOR SPACE 1225

Then the square of volumes $\left\{V_{1}^{3}(u, v, w)\right\}^{2}=b(u \times v, w)^{2}$ is calculated by

$$
\begin{aligned}
& \left\{V_{1}^{3}(u, v, w)\right\}^{2}=b(u \times v, w)^{2}=(\operatorname{det} A)^{2} \\
= & u_{1}^{2} v_{3}^{2} w_{2}^{2}+w_{1}^{2} u_{3}^{2} v_{2}^{2}+v_{1}^{2} u_{2}^{2} w_{3}^{2}+v_{1}^{2} u_{3}^{2} w_{2}^{2}+w_{1}^{2} u_{2}^{2} v_{3}^{2} \\
& +u_{1}^{2} v_{2}^{2} w_{3}^{2}-2 u_{1}^{2} v_{2} w_{3} v_{3} w_{2}-2 u_{1} v_{2} w_{3}^{2} v_{1} u_{2}+2 u_{1} v_{2} w_{3} v_{1} u_{3} w_{2} \\
& +2 u_{1} v_{2} w_{3} w_{1} u_{2} v_{3}-2 u_{1} v_{2}^{2} w_{3} w_{1} u_{3}+2 u_{1} v_{3} w_{2} v_{1} u_{2} w_{3}-2 u_{1} v_{3} w_{2}^{2} v_{1} u_{3} \\
& -2 u_{1} v_{3}^{2} w_{2} w_{1} u_{2}+2 u_{1} v_{3} w_{2} w_{1} u_{3} v_{2}-2 v_{1}^{2} u_{2} w_{3} u_{3} w_{2}-2 v_{1} u_{2}^{2} w_{3} w_{1} v_{3} \\
& +2 v_{1} u_{2} w_{3} w_{1} u_{3} v_{2}+2 v_{1} u_{3} w_{2} w_{1} u_{2} v_{3}-2 v_{1} u_{3}^{2} w_{2} w_{1} v_{2}-2 w_{1}^{2} u_{2} v_{3} u_{3} v_{2} .
\end{aligned}
$$

As we expected, we can see that

$$
\begin{aligned}
& \left|\begin{array}{ccc}
-b(u, u) & -b(u, v) & -b(u, w) \\
-b(v, u) & -b(v, v) & -b(v, w) \\
-b(w, u) & -b(w, v) & -b(w, w)
\end{array}\right| \\
= & u_{1}^{2} v_{3}^{2} w_{2}^{2}+w_{1}^{2} u_{3}^{2} v_{2}^{2}+v_{1}^{2} u_{2}^{2} w_{3}^{2}+v_{1}^{2} u_{3}^{2} w_{2}^{2}+w_{1}^{2} u_{2}^{2} v_{3}^{2} \\
& +u_{1}^{2} v_{2}^{2} w_{3}^{2}-2 u_{1}^{2} v_{2} w_{3} v_{3} w_{2}-2 u_{1} v_{2} w_{3}^{2} v_{1} u_{2} \\
& +2 u_{1} v_{2} w_{3} v_{1} u_{3} w_{2}+2 u_{1} v_{2} w_{3} w_{1} u_{2} v_{3} \\
& -2 u_{1} v_{2}^{2} w_{3} w_{1} u_{3}+2 u_{1} v_{3} w_{2} v_{1} u_{2} w_{3} \\
& -2 u_{1} v_{3} w_{2}^{2} v_{1} u_{3}-2 u_{1} v_{3}^{2} w_{2} w_{1} u_{2} \\
& +2 u_{1} v_{3} w_{2} w_{1} u_{3} v_{2}-2 v_{1}^{2} u_{2} w_{3} u_{3} w_{2} \\
& -2 v_{1} u_{2}^{2} w_{3} w_{1} v_{3}+2 v_{1} u_{2} w_{3} w_{1} u_{3} v_{2} \\
& +2 v_{1} u_{3} w_{2} w_{1} u_{2} v_{3}-2 v_{1} u_{3}^{2} w_{2} w_{1} v_{2}-2 w_{1}^{2} u_{2} v_{3} u_{3} v_{2} .
\end{aligned}
$$

We can easily calculate $(3.3)$ and $(3,4)$ using the maple program. The maple program for calculating (3.3) and (3.4) are given by

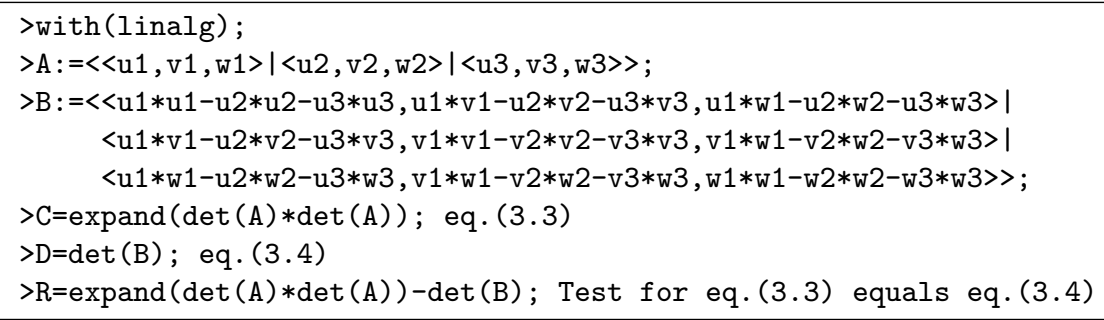

Hence we see that

$$
\left\{V_{1}^{3}(u, v, w)\right\}^{2}=\left|\begin{array}{lll}
-b(u, u) & -b(u, v) & -b(u, w) \\
-b(v, u) & -b(v, v) & -b(v, w) \\
-b(w, u) & -b(w, v) & -b(w, w)
\end{array}\right|
$$

from (3.3) and (3.4). 
Finally, let us investigate the volume $V_{1}^{n}\left(u_{1}, u_{2}, \ldots, u_{n}\right)$ of parallel $2 n$-hedron spanned by linearly independent timelike vectors $u_{1}, u_{2}, \ldots, u_{n}$ and none of them are in the same timecone. Then we have the following theorem.

Theorem 3.2. Let $u_{1}, u_{2}, \ldots, u_{n}$ be linearly independent timelike vectors in $U_{1}^{n}$ and none of them are in the same timecone. Then the volume of parallel $2 n$-hedron spanned by $u_{1}, u_{2}, \ldots, u_{n}$ is

$$
\begin{aligned}
& \left\{V_{1}^{n}\left(u_{1}, u_{2}, \ldots, u_{n}\right)\right\}^{2} \\
= & \left|\begin{array}{cccc}
-b\left(u_{1}, u_{1}\right) & -b\left(u_{1}, u_{2}\right) & \ldots & -b\left(u_{1}, u_{n}\right) \\
-b\left(u_{2}, u_{1}\right) & -b\left(u_{2}, u_{2}\right) & \ldots & -b\left(u_{2}, u_{n}\right) \\
\vdots & \vdots & \ddots & \vdots \\
-b\left(u_{n}, u_{1}\right) & -b\left(u_{n}, u_{2}\right) & \ldots & -b\left(u_{n}, u_{n}\right)
\end{array}\right| .
\end{aligned}
$$

Proof. For $V_{1}^{n}\left(u_{1}, u_{2}, \ldots, u_{n}\right)$, we have formulated in (3.1), (3.2) and Theorem 3.1 for $n=1,2,3$. Assume that $V_{1}^{n-1}\left(u_{1}, u_{2}, \ldots, u_{n-1}\right)$ is given by

$$
\begin{aligned}
&\left\{V_{1}^{n-1}\left(u_{1}, u_{2}, \ldots, u_{n-1}\right)\right\}^{2} \\
&=\left|\begin{array}{cccc}
-b\left(u_{1}, u_{1}\right) & -b\left(u_{1}, u_{2}\right) & \ldots & -b\left(u_{1}, u_{n-1}\right) \\
-b\left(u_{2}, u_{1}\right) & -b\left(u_{2}, u_{2}\right) & \cdots & -b\left(u_{2}, u_{n-1}\right) \\
\vdots & \vdots & \ddots & \vdots \\
-b\left(u_{n-1}, u_{1}\right) & -b\left(u_{n-1}, u_{2}\right) & \cdots & -b\left(u_{n-1}, u_{n-1}\right)
\end{array}\right|
\end{aligned}
$$

and consider the volume of parallel $2 n$-hedron spanned by linearly independent timelike vectors $u_{1}, u_{2}, \ldots, u_{n}$. Let $v_{n}=u_{n}-\sum_{i=1}^{n-1} \lambda_{i} u_{i}$ for $\lambda_{1}, \lambda_{2}, \ldots, \lambda_{n-1} \in$ $\mathbb{R}$. Then we can take $\lambda_{1}, \lambda_{2}, \ldots, \lambda_{n-1}$ such that

$$
b\left(v_{n}, u_{j}\right)=b\left(u_{n}, u_{j}\right)-\sum_{i=1}^{n-1} \lambda_{i} b\left(u_{i}, u_{j}\right)=0
$$

for $j=1,2, \ldots, n-1$. In this case, $w_{n}=\sum_{i=1}^{n-1} \lambda_{i} u_{i}$ is the projection of $u_{n}$ to the $(n-1)$-dimensional vector space $W_{1}^{n-1}$ spanned by $u_{1}, u_{2}, \ldots, u_{n-1}$, and $v_{n}=u_{n}-w_{n}$ is the component of $u_{n}$ to $\left(W_{1}^{n-1}\right)^{\perp}$.

Therefore, by induction,

$$
V_{1}^{n}\left(u_{1}, u_{2}, \ldots, u_{n}\right)=V_{1}^{n-1}\left(u_{1}, u_{2}, \ldots, u_{n-1}\right) \times\left\|v_{n}\right\| .
$$

Whereas, in the right hand side of (3.5), if we subtract the sum of $\sum_{i=1}^{n-1} \lambda_{i} \times($ component of $i$-th column $)=\sum_{i=1}^{n-1} \lambda_{i}\left(-b\left(u_{j}, u_{i}\right)\right)(j=1,2, \ldots, n-1)$, 
from each component of the $n$-th column, then we get

$$
\begin{aligned}
& \left|\begin{array}{cccc}
-b\left(u_{1}, u_{1}\right) & -b\left(u_{1}, u_{2}\right) & \ldots & -b\left(u_{1}, u_{n}\right) \\
-b\left(u_{2}, u_{1}\right) & -b\left(u_{2}, u_{2}\right) & \cdots & -b\left(u_{2}, u_{n}\right) \\
\vdots & \vdots & \ddots & \vdots \\
-b\left(u_{n}, u_{1}\right) & -b\left(u_{n}, u_{2}\right) & \cdots & -b\left(u_{n}, u_{n}\right)
\end{array}\right| \\
= & \left|\begin{array}{cccc}
-b\left(u_{1}, u_{1}\right) & \ldots & -b\left(u_{1}, u_{n-1}\right) & 0 \\
\vdots & \vdots & \ddots & \vdots \\
-b\left(u_{n-1}, u_{1}\right) & \ldots & -b\left(u_{n-1}, u_{n-1}\right) & 0 \\
-b\left(u_{n}, u_{1}\right) & \ldots & -b\left(u_{n}, u_{n-1}\right) & -b\left(u_{n}, v_{n}\right)
\end{array}\right| \\
= & \left\{V_{1}^{n-1}\left(u_{1}, u_{2}, \ldots, u_{n-1}\right)\right\}^{2} \times\left|-b\left(u_{n}, v_{n}\right)\right| .
\end{aligned}
$$

Since $b\left(v_{n}, u_{j}\right)=0(j=1,2, \ldots, n-1)$ by $(3.6)$, we obtain

$$
\begin{aligned}
b\left(v_{n}, v_{n}\right) & =b\left(u_{n}, v_{n}\right)-\sum_{i=1}^{n-1} \lambda_{i} b\left(u_{i}, v_{n}\right) \\
& =b\left(u_{n}, v_{n}\right) .
\end{aligned}
$$

Hence, by (3.7) and (3.8), we can see that

$$
\begin{aligned}
& \left|\begin{array}{cccc}
-b\left(u_{1}, u_{1}\right) & -b\left(u_{1}, u_{2}\right) & \cdots & -b\left(u_{1}, u_{n}\right) \\
-b\left(u_{2}, u_{1}\right) & -b\left(u_{2}, u_{2}\right) & \cdots & -b\left(u_{2}, u_{n}\right) \\
\vdots & \vdots & \ddots & \vdots \\
-b\left(u_{n}, u_{1}\right) & -b\left(u_{n}, u_{2}\right) & \cdots & -b\left(u_{n}, u_{n}\right)
\end{array}\right| \\
= & \left\{V_{1}^{n-1}\left(u_{1}, u_{2}, \ldots, u_{n-1}\right)\right\}^{2} \times\left\|v_{n}\right\|^{2} \\
= & \left\{V_{1}^{n}\left(u_{1}, u_{2}, \ldots, u_{n}\right)\right\}^{2} .
\end{aligned}
$$

Thus the proof is completed.

Since the orthogonality of timelike vectors induces a linearly independence and none of them are in the same timecone by the Lemma 2.1, we have:

Corollary 3.3. Let $u_{1}, u_{2}, \ldots, u_{n}$ be mutually orthogonal timelike vectors in $U_{1}^{n}$. Then we have the same formula as (3.5):

$$
\left\{V_{1}^{n}\left(u_{1}, u_{2}, \ldots, u_{n}\right)\right\}^{2}=\left|\begin{array}{cccc}
-b\left(u_{1}, u_{1}\right) & -b\left(u_{1}, u_{2}\right) & \cdots & -b\left(u_{1}, u_{n}\right) \\
-b\left(u_{2}, u_{1}\right) & -b\left(u_{2}, u_{2}\right) & \cdots & -b\left(u_{2}, u_{n}\right) \\
\vdots & \vdots & \ddots & \vdots \\
-b\left(u_{n}, u_{1}\right) & -b\left(u_{n}, u_{2}\right) & \cdots & -b\left(u_{n}, u_{n}\right)
\end{array}\right|
$$

\section{References}

[1] J. K. Beem, P. E. Ehrlich, and K. L. Easley, Global Lorentzian Geometry, Second edition. Monographs and Textbooks in Pure and Applied Mathematics, 202. Marcel Dekker, Inc., New York, 1996. 
[2] U-Hang Ki and J. S. Pak, Projective Geometry, Hak-Moon Sa Press, Seoul, 1982.

[3] H. Nakagawa, J.-H. Kwon, and Y.-S. Pyo, Introduction to Semi-Riemannian geometry, Kyung Moon Publishers, Seoul, 2000.

[4] B. O'Neill, Semi-Riemannian Geometry, Pure and Applied Mathematics, 103, Academic Press, Inc. [Harcourt Brace Jovanovich, Publishers], New York, 1983.

SEONGIL PARK

Department of Applied Mathematics

KYUNG HEE UNIVERSITY

YONGIN 446-701, KoreA

E-mail address: sipark@khu.ac.kr

BYUNG HAK KIM

Department of Applied Mathematics

KyUng HeE University

YONGIN 446-701, KoreA

E-mail address: bhkim@khu.ac.kr

Jin Hyuk ChOI

College of Liberal Arts

KYUNG HeE UnIVERSity

YONGIN 446-701, KoREA

E-mail address: jinhchoi@khu.ac.kr

Young OK Lee

Department of Mathematics

KYUNG HeE University

YONGIN 446-701, KoREA

E-mail address: ylee6604@korea.com 